\title{
BMJ Open Long-term observational approach in women with histological diagnosis of cervical low-grade squamous intraepithelial lesion: an Italian multicentric retrospective cohort study
}

Andrea Ciavattini, ${ }^{1}$ Matteo Serri, ${ }^{1}$ Jacopo Di Giuseppe, ${ }^{1}$ Carlo Antonio Liverani, ${ }^{2}$ Barbara Gardella, ${ }^{3}$ Maria Papiccio, ${ }^{1}$ Giovanni Delli Carpini, ${ }^{1}$ Stefano Morini, ${ }^{1}$ Nicolò Clemente, ${ }^{4}$ Francesco Sopracordevole ${ }^{4}$

To cite: Ciavattini A, Serri M, Di Giuseppe J, et al. Long-term observational approach in women with histological diagnosis of cervical low-grade squamous intraepithelial lesion: an Italian multicentric retrospective cohort study. BMJ Open 2019;9:e024920. doi:10.1136/ bmjopen-2018-024920

- Prepublication history for this paper is available online To view these files, please visit the journal online (http://dx.doi. org/10.1136/bmjopen-2018024920).

Received 20 June 2018 Revised 25 February 2019 Accepted 28 May 2019
Check for updates

(C) Author(s) (or their employer(s)) 2019. Re-use permitted under CC BY-NC. No commercial re-use. See rights and permissions. Published by BMJ.

For numbered affiliations see end of article.

Correspondence to Professor Andrea Ciavattini; ciavattini.a@libero.it

\section{ABSTRACT}

Objective To evaluate the risk of progression to highgrade squamous intraepithelial lesion (HSIL) (CIN2-3) or invasive cancer in women with histopathological diagnosis of low-grade squamous intraepithelial lesion (LSIL) (CIN1), managed in a long-term observational approach up to 5 years.

Design Retrospective cohort study.

Setting Four tertiary referral hospital.

Participants 434 women with adequate colposcopy and complete colposcopic charts were included in the present analysis. Women with glandular lesions on the referral cytology or previous diagnosis of cervical dysplasia or invasive cervical cancer or with synchronous vaginal, or with HIV infection or immunodepression were excluded.

Primary and secondary outcome measures The main study outcome was the rate of progression to histopathological HSIL (CIN2-3) or invasive cancer at any time during 5 years of follow-up. The possible risk factors were also evaluated. As secondary outcome, we analysed the possible risk factors at the 24-month evaluation for histopathological HSIL (CIN2-3) or invasive cancer progression between 2 and 5 years from initial diagnosis.

Results A progression to histopathological HSIL (CIN2-3) was found in a total of $32(7.4 \%)$ cases during 5 years of follow-up. A histopathological diagnosis of HSIL (CIN3) was found in four patients $(0.9 \%)$ and no case of invasive cancer was detected. High-grade cytology at inclusion and the presence of a positive high-risk human papillomavirus (HR-HPV) DNA test at 2 years from inclusion maintained a significant correlation with the risk of histopathological progression to HSIL (CIN2-3).

Conclusions The results of our study showed a low rate (7.4\%) of histopathological progression to HSIL (CIN2-3) in women with LSIL (CIN1) diagnosis during long-term follow-up up to 5 years. In case of positive HR-HPV DNA test at the 2 years evaluation an excisional treatment could be the preferred choice to prevent progression to HSIL (CIN2-3) in the following years, preferring a continuation of follow-up in case of HR-HPV DNA negative result.

\section{Strengths and limitations of this study}

- This is a large cohort study of 434 women with histological diagnosis of LSIL (CIN1) with a long-term 5 -year follow-up.

- The study can help us to find a possible subgroup of women at higher risk of progression or persistence of LSIL (CIN1).

- All patients were managed by certified expert operators in the four involved centres.

- All the cervical cytology and histological samples were analysed by cytologists and pathologists of the tertiary care institutions involved, with particular expertise in the field of cervical intraepithelial and invasive lesions.

- Our study is a multicentric retrospective study, which might have introduced a selection bias.

\section{INTRODUCTION}

The cervical low-grade squamous intraepithelial lesion (LSIL) (CIN1), defined according to the 2012 revised Lower Anogenital Squamous Terminology (LAST) terminology, ${ }^{1}$ can be considered as the transient expression of a self-limited human papillomavirus (HPV) infection. Since LSIL (CIN1) does not determine a significant higher risk for high-grade lesions or cervical cancer, it does not represent a target for cervical cancer screening. ${ }^{2}$ Moreover, considering that LSIL (CIN1) is characterised by a low rate of progression to high-grade lesions ${ }^{3-5}$ and by a high rate of spontaneous regression (up to $60 \%-80 \%$ of all cases within $2-5$ years $\left.{ }^{6-9}\right)$, especially in young women, ${ }^{10}{ }^{11}$ a conservative approach with follow-up should be preferred in the first instance. ${ }^{12-15}$ However, even if the currently available guidelines provide the possibility both to treat or continue follow-up if LSIL 
(CIN1) persist for at least 2 years, ${ }^{13}$ there is no consensus on the optimal management in case of long-term follow-up without treatment, on the safety of prolonging follow-up beyond 2 years from initial diagnosis, and on the overall duration of a long-term follow-up.

The safety of a long-term follow-up should be based on risk for developing high-grade squamous intraepithelial lesion (HSIL) (CIN3) or invasive cancer, since this risk is considered to be the better indicator for reducing cancer incidence. $^{2}$ It is reported that an HSIL (CIN3) or invasive cancer risk lower than $2.0 \%$ is considered acceptable to recommend screening in 5 years, while a risk between $2.0 \%$ and $10.0 \%$ might warrant rescreening in a year. ${ }^{2}$ Nevertheless, in the clinical practice, HSIL (CIN2-3) or invasive cancer is the threshold for treatment ${ }^{2}$ and this risk should guide the design of a long-term follow-up.

The aim of this study was therefore to evaluate the risk of progression to HSIL (CIN2-3) or invasive cancer in women with histopathological diagnosis of LSIL (CIN1), managed in a long-term observational approach up to 5 years. As secondary aim, we investigated the potential risk factors at 2 years from initial diagnosis for progression to HSIL (CIN2-3) or invasive cancer in the following 3 years in women with low-grade cytology without histopathological assessment at colposcopy or histopathological LSIL (CIN1) diagnosis, in order to identify those patients that may warrant treatment after 2 years instead of prolonged follow-up.

\section{MATERIALS AND METHODS}

All the women older than 21 years with a histopathological diagnosis of LSIL (CIN1) performed from January 2006 to December 2011 were considered. All patients underwent colposcopy-guided biopsy of suspicious areas on the uterine cervix after an abnormal referral cervical cytology and subsequent colposcopy, according to current guidelines. ${ }^{1315}$

Women with previous diagnosis or treatment for cervical dysplasia or invasive cervical cancer were excluded, as well as women with synchronous vaginal intraepithelial lesions or vaginal cancer and women with HIV infection or immunodepression.

Patients were identified by searching our clinical databases, and the medical records of women fulfilling the study inclusion and exclusion criteria were retrospectively analysed in an observational cohort study.

We collected data regarding the referral cytology, the colposcopic examination and the histopathological findings on biopsy. Moreover, we collected pertinent sociodemographic and clinical data about age, menopausal status, oral contraceptive (OC) use and tobacco use.

All the cervical cytology and histological samples were analysed by cytologists and pathologists of the involved Institutions, with particular expertise in the field of cervical intraepithelial and invasive lesions. Similarly, all the colposcopies (and the colposcopy-guided biopsies) were performed by experienced gynaecologists of the involved institutions certified by Italian Society of Colposcopy and Cervico-Vaginal Pathology (SICPCV) certification.

Cytological abnormalities were classified, according to the Bethesda system terminology, ${ }^{5}$ in atypical squamous cells of undetermined significance (ASC-US), LSIL, atypical squamous cells, cannot rule out high-grade SIL (ASC-H) and HSIL. A triage test with high-risk human papillomavirus (HR-HPV) DNA was performed at inclusion in women with ASC-US cytology, referring to colposcopy only those who tested positive. ASC-US and LSIL were categorised as 'low-grade cytology' while HSIL and ASC-H were categorised as 'high-grade cytology'. Women with glandular lesions on the referral cytology were excluded.

Colposcopic examinations were recorded accordingly to the 2011 revised colposcopic terminology of the International Federation for Cervical Pathology and Colposcopy (IFCPC).${ }^{16}$ In particular, the abnormal colposcopic findings of 'flat acetowhite epithelium', 'fine mosaic', 'fine punctation', 'iodine partial positivity' were considered as 'grade 1 colposcopic findings' (ANTZG1) and the abnormal colposcopic findings of 'dense acetowhite epithelium', 'coarse mosaic', 'coarse punctation' and 'iodine negativity' were considered as 'grade 2 colposcopic findings' (ANTZG2). The evidence of 'atypical vessels', 'irregular surface', 'erosion', 'ulceration' or 'wide irregular punctation and mosaic' was considered as suspicious features for invasive cancer. The colposcopies performed before the introduction of the 2011 IFCPC terminology were revised accordingly, through the revision of colposcopic charts and images. Only women with adequate colposcopy and complete colposcopic charts were included in the present analysis.

After reviewing the slides, the histopathologic results were reported according to LAST terminology for HPV-associated lesions and defined as LSIL (CIN1), HSIL $\left(\right.$ CIN2-3) ${ }^{1}$ or invasive cancer.

After the initial histopathological diagnosis of LSIL (CIN1), all patients underwent scheduled follow-up with repeat cytology at 12 and 24 months from initial diagnosis, and immediate referral to colposcopy in case of positive cytology of any grade. In addition, an HR-HPV DNA test was performed at 24 months from inclusion. All women who presented negative cytology results and negative HR-HPV DNA test at 24 months were sent to routine screening with repeat cytology at 3 years (5 years from initial diagnosis). At 24 months, in the case of low-grade cytology without histopathological assessment after colposcopy or histopathological LSIL (CIN1) diagnosis, a repeat cytology was scheduled every 12 months up to 5 years from initial diagnosis with immediate referral to colposcopy in case of positive results. At any time, in the case of histopathological HSIL (CIN2-3) diagnosis, patients were subjected to cervical excision procedure. At 5 years from initial diagnosis, in all women with low-grade cytology without histopathological assessment after colposcopy or 
histopathological CIN1 diagnosis, a cervical excision procedure was performed.

The main study outcome was the rate of progression to histopathological HSIL (CIN2-3) or invasive cancer at any time during 5 years of follow-up after the first diagnosis of LSIL (CIN1). The possible risk factors at inclusion (age, tobacco use, menopause, OC use, cytology results and colposcopic appearance) associated with histopathological HSIL (CIN2-3) or invasive cancer progression were also evaluated. The rate of progression to HSIL (CIN3) was also recorded.

As secondary outcome, we analysed the possible risk factors at the 24-month evaluation (age, tobacco use, menopause, OC use, cytology results, HR-HPV DNA results and colposcopic appearance) for histopathological HSIL (CIN2-3) or invasive cancer progression between 2 and 5 years from initial diagnosis in women with low-grade cytology without histopathological assessment after colposcopy or histopathological LSIL (CIN1) diagnosis, in order to identify a subgroup of those patients in which a cervical excision at 24 months from initial diagnosis could be more appropriate than a prolonged follow-up.

Statistical analysis was performed using IBM SPSS V.22.0 (IBM Corporation).

The sample size was determined considering the rate of progression to HSIL (CIN2-3) in 5 years of follow-up without treatment in women with histopathological diagnosis of LSIL (CIN1) as the primary outcome measure; data from previous literature reported a rate of progression to HSIL (CIN2-3) of $10 \%-15 \%{ }^{12}{ }^{17}$ Setting a $6 \%$ wide $95 \%$ CI (with alpha $=0.05$ and beta $=0.20$ ) and an estimated dropout rate of $20 \%$, the determined minimum sample size was 416 women. Therefore, we retrospectively included patients from the databases of the four involved institutions, starting from year 2011 until reaching at least 416 cases.

$\chi^{2}$ testing and $t$-test were used, as appropriate, for comparison between dichotomous and continuous variables in the bivariate analysis. A multivariate logistic regression was used to identify possible risk factors associated with histopathological HSIL (CIN2-3) or invasive cancer progression both at 5 years of follow-up and at 2 years of follow-up, in the entire study cohort or in women with low-grade cytology without histopathological assessment after colposcopy or histopathological LSIL (CIN1) diagnosis, respectively. Only those variables that were significant in the bivariate analysis were considered for the logistic regression model. A Kaplan-Meier survival curve was constructed to determine the time-based rate of progression to histopathological HSIL (CIN2-3). A $\mathrm{p}<0.05$ was considered statistically significant. A written informed consent for use of personal data was obtained from each woman.

\section{Patient and public involvement}

We did not directly include patient and public involvement PPI in this study, but the database used in the study was developed with PPI and is updated by a committee that includes patient representatives.

\section{RESULTS}

During the study period, 434 women, fulfilling the study inclusion and exclusion criteria, constituted the study cohort.

The mean age was 35.0 years (range: $23.0-52.0$ ), 114 women $(26.3 \%)$ were tobacco users, $151(34.9 \%)$ used OC and 46 women (10.6\%) were in menopause.

The referral cervical cytology was 'low-grade' in 398 women $(91.7 \%)$ and 'high-grade' in the remaining 36 women $(8.3 \%)$. The colposcopic findings were: ANTZG1 in 399 cases (91.9\%) and ANTZG2 in 35 women $(8.1 \%)$.

In the whole study cohort, a progression to histopathological HSIL (CIN2-3) was found in a total of $32(7.4 \%)$ cases during 5 years of follow-up. A histopathological diagnosis of HSIL (CIN3) was found in four patients $(0.9 \%)$ and no case of invasive cancer was detected.

Among the $402(92.6 \%)$ women who did not present a histopathological progression to HSIL (CIN2-3) or invasive cancer, 6 (1.4\%) showed a low-grade cytology without histopathological assessment at colposcopy or LSIL (CIN1) diagnosis after 5 years from inclusion and were subjected to cervical excision. The final histopathological diagnosis was LSIL (CIN1) in four cases (66.7\%) and negative for dysplasia in two cases (33.3\%) (figure 1).

The bivariate analysis of possible risk factors for histopathological progression to HSIL during 5 years of follow-up is reported in table 1 . Among the factors that were significantly associated with progression at bivariate (tobacco use, ANTZG2 at inclusion and high-grade cytology at inclusion), only high-grade cytology at inclusion maintained a significant correlation with the risk of histopathological progression to HSIL (CIN2-3) $(36.1 \%$ vs $4.8 \%$ ), with an OR of $0.1,95 \%$ CI (0.04 to 0.23$)$, $\mathrm{p}<0.0001$.

Figure 2 represents the Kaplan-Meier curves for 5-year probability of progression to histopathological HSIL according to referral cervical cytology at initial histopathological diagnosis of LSIL (CIN1). Women with initial low grade cytology presented a significantly higher probability of progression to histopathological HSIL during 5 years with respect to high-grade cytology $(\mathrm{p}<0.001)$.

At 2years from inclusion, 354 (81.6\%) women presented a negative cervical cytology, $67(15.4 \%)$ a low-grade cytology without histopathological assessment after colposcopy or CIN1 histopathological diagnosis, and $13(3.0 \%)$ a histopathological progression to HSIL. Among those patients, two $(0.5 \%)$ presented a histopathological progression to HSIL (CIN3), with no case of invasive cancer (figure 1).

A total of 19 (4.4\%) women showed a histopathological progression to HSIL between 2 and 5 years of follow-up, with $2(0.5 \%)$ cases of progression to HSIL (CIN3) and no case of invasive cancer. 


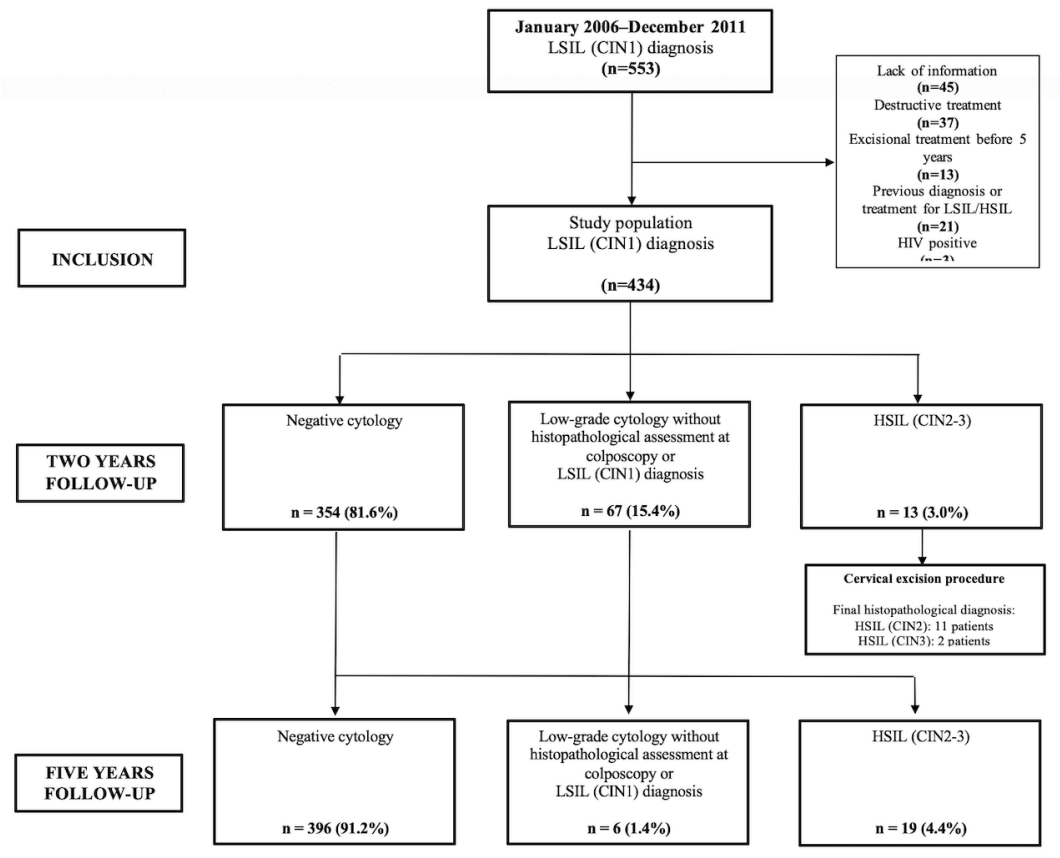

Figure 1 Flowchart of study population and follow-up.

All women who resulted negative at the 2-year cervical cytological assessment showed a negative cytology after 3 years.

Among the 67 women who presented a low-grade cytology without histopathological assessment after colposcopy or LSIL (CIN1) histopathological diagnosis at 2 years from inclusion, $19(28.4 \%)$ progressed to HSIL (CIN2-3) between 2 and 5 years.

Table 2 reports the bivariate analysis of the risk factors at the 2-year evaluation for histopathological progression to HSIL between 2 and 5 years of follow-up in women with a low-grade cytology without histopathological assessment after colposcopy or LSIL (CIN1) histopathological diagnosis. Only the presence of a positive HR-HPV DNA test at 2 years from inclusion was associated with a higher risk of histopathological progression to HSIL between 2 and 5 years of follow-up, with an OR of 5.6 (95\% CI 1.7 to 18.3, $\mathrm{p}=0.002$ ).

\section{DISCUSSION}

The results of our study showed a low rate $(7.4 \%)$ of histopathological progression to HSIL (CIN2-3) in women with LSIL (CIN1) diagnosis during long-term follow-up up to 5 years. The risk of HSIL (CIN3) was $0.9 \%$ and no case of invasive cancer was found. A high-grade referral cytology was associated with a higher risk of progression to histopathological HSIL ( $36.1 \%$ vs $4.8 \%$ ), with an OR of $0.1,95 \%$ CI ( 0.04 to 0.23$), \mathrm{p}<0.0001$.

These findings are consistent with existing literature: the study from Katki et $a l^{17}$ showed a risk of progression to CIN2+ atfive years of $10.0 \%$ in the case of low-grade cytology at inclusion and of $24.0 \%$ in the case of highgrade cytology; instead, the risk of CIN3+ is reported to be between $3.8 \%$ and $15.0 \%$ according to referral cytology. ${ }^{17}$ The risk of invasive cancer is instead lower $(0.2 \%-2.1 \%)^{17}$ ; in a Norwegian study of 520 women with a negative

Table 1 Bivariate analysis of possible risk factors at inclusion for progression to histopathological HSIL (CIN2-3) during 5 years of follow-up in the study cohort $(n=434)$

\begin{tabular}{|c|c|c|c|c|}
\hline Characteristics & $\begin{array}{l}\text { Whole study cohort } \\
(n=434)\end{array}$ & $\begin{array}{l}\text { No progression } \\
(n=402)\end{array}$ & $\begin{array}{l}\text { Progression to HSIL } \\
(n=32)\end{array}$ & $\mathbf{P}$ \\
\hline Age (years) & $35.7 \pm 9.6$ & $35.8 \pm 9.6$ & $33.9 \pm 9.2$ & 0.28 \\
\hline Tobacco use (\%) & $117(27.0 \%)$ & $102(25.4)$ & $15(46.9)$ & 0.01 \\
\hline Menopause (\%) & $46(10.6 \%)$ & $44(10.9)$ & $2(6.3)$ & 0.61 \\
\hline OC use (\%) & $121(27.9)$ & $108(26.9)$ & $13(40.6)$ & 0.15 \\
\hline High-grade cytology at inclusion (\%) & $36(8.3)$ & $23(5.7)$ & $13(43.8)$ & $<0.0001$ \\
\hline ANTZG2 at inclusion (\%) & $36(8.3)$ & $29(7.2)$ & $7(21.9)$ & 0.01 \\
\hline
\end{tabular}

Data are mean \pm SD or $\mathrm{n}(\%)$ as appropriate.

ANTZG2, grade 2 colposcopic findings; HSIL, high-grade squamous intraepithelial lesion. 


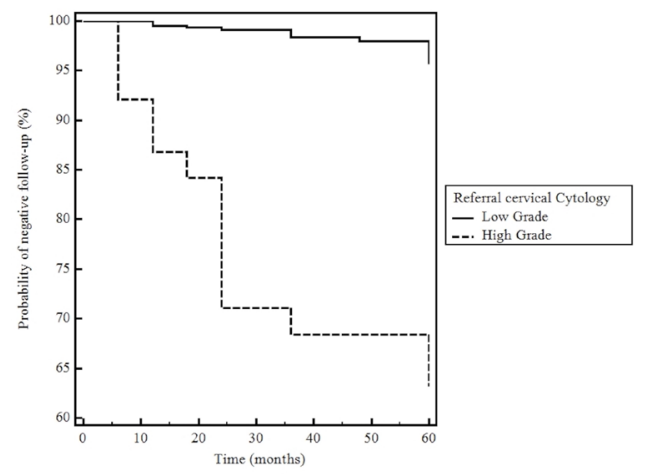

Figure 2 Kaplan-Meier analysis of time-based rate of progression to histopathological high-grade squamous intraepithelial lesion (CIN2-3) on the basis of the referral initial cytology.

cervical biopsy after ASC-H/HSIL cytology or ASC-US/ LSIL cytology with a positive 5-type HPV mRNA test, seven women $(1.3 \%)$ developed invasive cervical cancer in the follow-up. ${ }^{18}$

Even if the risk of developing CIN3+ is considered to be the better indicator for reducing cancer incidence, ${ }^{2}$ the goal of clinical management is to identify and treat high-grade disease ${ }^{1}$ (HSIL according to LAST terminology), which can also include CIN2 lesions, that is thought to represent a mixture of low-grade and precancerous disease, ${ }^{1}$ with an intermediate risk of progression. Moreover, current clinical guidelines recommend treatment of both CIN2 and CIN3 lesions, with the exception of special patient categories. ${ }^{13} 15$ For these reasons, when evaluating the best clinical management and safety of a prolonged follow-up of LSIL (CIN1) lesions, the risk of

Table 2 Bivariate analysis of the risk factors at the 2-year evaluation for histopathological progression to HSIL (CIN23) between 2 and 5 years of follow-up in women with a low-grade cytology without histopathological assessment after colposcopy or LSIL (CIN1) histopathological diagnosis $(n=67)$

\begin{tabular}{llll}
\hline Characteristics & $\begin{array}{l}\text { No progression } \\
(\mathbf{n = 4 8 )}\end{array}$ & $\begin{array}{l}\text { Progression } \\
(\mathbf{n = 1 9 )}\end{array}$ & $\mathbf{P}$ \\
\hline Age (years) & $33.4 \pm 9.9$ & $34.4 \pm 10.7$ & 1.00 \\
\hline Tobacco use (\%) & $22(45.8 \%)$ & $8(42.1 \%)$ & 0.99 \\
\hline Menopause (\%) & $5(10.4 \%)$ & $1(5.3 \%)$ & 0.85 \\
\hline OC use (\%) & $14(29.2 \%)$ & $4(21.0 \%)$ & 0.71 \\
\hline HSIL at inclusion (\%) & $0(-)$ & $0(-)$ & - \\
\hline ANTZG2 at inclusion (\%) & $0(-)$ & $0(-)$ & - \\
\hline ANTZG2 at 2years (\%) & $3(6.3 \%)$ & $3(15.8 \%)$ & 0.45 \\
\hline HSIL at 2years (\%) & $0(-)$ & $2(10.5 \%)$ & 0.14 \\
\hline Positive HR-HPV DNA at & $16(33.3 \%)$ & $14(73.7 \%)$ & 0.006 \\
2years (\%) & & & \\
\hline
\end{tabular}

Data are mean \pm SD or $\mathrm{n}(\%)$ as appropriate.

ANTZG2, grade 2 colposcopic findings; HR-HPV, high-risk human papillomavirus; HSIL, high-grade squamous intraepithelial lesion;

LSIL, low-grade squamous intraepithelial lesion. progression to HSIL (CIN2-3) or invasive cancer should be considered, rather than the risk of progression to CIN3+. Our data support the opportunity of a prolonged follow-up, taking also into account that only $0.9 \%$ on included patient developed a CIN3 and none an invasive cancer during 5 years of observational approach, below the threshold of $2.0 \%$ that is considered acceptable to recommend screening in 5 years.

In our series, patients that manifest a low-grade cytology without histopathological assessment after colposcopy or CIN1 histopathological diagnosis at 2years from initial diagnosis of LSIL (CIN1) had a risk of $28.4 \%$ of developing HSIL (CIN2-3) in the following 3 years. In order to safely extend follow-up and to avoid unnecessary treatments, we investigated the risk factors associated with progression that could identify patients where treatment could be more appropriate. Among the considered factors, only women who resulted HR-HPV DNA positive at the 2-year evaluation presented a six-time higher risk of progression to HSIL in the following 3 years with respect to HR-HPV DNA negative women. Therefore, in this subgroup of women, an excisional treatment at 2 years could be the preferred choice to prevent progression to HSIL (CIN2-3) in the following years, preferring a continuation of follow-up in case of HR-HPV DNA negative result. However, since different HPV subtypes may determine different risks of high-grade lesions (up to a risk of $47.4 \%$ in case of HPV 16 2-year persistence), ${ }^{19}$ a potential bias of the present study is the absence of HPV typing. In contrast to existing literature, ${ }^{12}{ }^{17}$ we did not find a correlation between cervical cytology at inclusion or at 2 years and risk of progression to histopathological HSIL between two and 5 years, but only with HR-HPV DNA positivity at 2 years.

Strengths of our study are the multicentric nature and the large cohort of patients and the certified expertise of operators in the four involved centres.

Limitations are the retrospective nature which might have introduced a selection bias, the absence of HPV typing and the absence of histopathological samples in every case of colposcopic evaluation.

\section{CONCLUSIONS}

Women with LSIL (CIN1) diagnosis presented a low rate of progression to HSIL (CIN2-3) during 5 years of follow-up, with higher risk in case of high-grade cytology at inclusion. These findings support a long-term observational approach up to 5 years in this subgroup of women avoiding unnecessary treatment, particularly useful in those women with desire for pregnancy, since an excisional treatment could potentially determine a higher risk of obstetric adverse events. ${ }^{20-24}$

After 2years of follow-up, in women with persistent low-grade cytology or with persistent histopathological diagnosis of LSIL (CIN1), an HR-HPV positive test may be helpful to identify those patients who will benefit from excisional treatment. Future studies about the usefulness 
of HPV typing or molecular biomarkers in stratifying the risk of progression are however needed.

\section{Author affiliations}

${ }^{1}$ Department of Odontostomatologic and Specialized Clinical Sciences, Università Politecnica delle Marche, Ancona, Italy

${ }^{2}$ Department of Mother and Infant Sciences, Università degli Studi di Milano, Milano, Italy

${ }^{3}$ Department of Clinical, Surgical, Diagnostic and Paediatric Sciences, Università degli Studi di Pavia, Pavia, Italy

${ }^{4}$ Gynecological Oncology Unit, Centro di Riferimento Oncologico, Aviano, Italy

Contributors JDG, MS, GDC and FS contributed to acquisition of data, statistical analysis, interpretation of data and manuscript writing. CAL, BG, MP, NC and SM contributed to acquisition and interpretation of data. $A C$ and FS supervised the project. AC, MS and JDG wrote the manuscript. All authors approved the final version to be published. AC is the guarantor of this work.

Funding The authors have not declared a specific grant for this research from any funding agency in the public, commercial or not-for-profit sectors.

Competing interests None declared.

Patient consent for publication Obtained.

Ethics approval Ethics approval for the review of case records was obtained from the local ethic committee of the Oncological Referral Center of Aviano (Pordenone, Italy) for this study (CRO-IRB 2014-08)

Provenance and peer review Not commissioned; externally peer reviewed.

Data sharing statement No additional data are available.

Open access This is an open access article distributed in accordance with the Creative Commons Attribution Non Commercial (CC BY-NC 4.0) license, which permits others to distribute, remix, adapt, build upon this work non-commercially, and license their derivative works on different terms, provided the original work is properly cited, appropriate credit is given, any changes made indicated, and the use is non-commercial. See: http://creativecommons.org/licenses/by-nc/4.0/.

\section{REFERENCES}

1. Darragh TM, Colgan TJ, Cox JT, et al. The Lower Anogenital Squamous Terminology Standardization Project for HPV-Associated Lesions: background and consensus recommendations from the College of American Pathologists and the American Society for Colposcopy and Cervical Pathology. J Low Genit Tract Dis 2012;16:205-42.

2. Castle PE, Sideri M, Jeronimo J, et al. Risk assessment to guide the prevention of cervical cancer. Am J Obstet Gynecol 2007;197(4):356. e1-356.e6.

3. Ciavattini A, Clemente N, Tsiroglou D, et al. Follow up in women with biopsy diagnosis of cervical low-grade squamous intraepithelial lesion (LSIL): how long should it be? Arch Gynecol Obstet 2017;295:997-1003.

4. Jordan J, Martin-Hirsch P, Arbyn M, et al. European guidelines for clinical management of abnormal cervical cytology, part 2. Cytopathology 2009;20:5-16.

5. Nayar R, Wilbur DC. The Pap Test and Bethesda 2014. J Low Genit Tract Dis 2015;19:175-84.

6. Petry KU. Management options for cervical intraepithelial neoplasia. Best Pract Res Clin Obstet Gynaecol 2011;25:641-51.
7. Prendiville W. The treatment of $\mathrm{CIN}$ : what are the risks? Cytopathology 2009;20:145-53.

8. Moscicki AB, Shiboski S, Hills NK, et al. Regression of lowgrade squamous intra-epithelial lesions in young women. Lancet 2004;364:1678-83.

9. Stoler MH, Schiffman M. Atypical Squamous Cells of Undetermined Significance-Low-grade Squamous Intraepithelial Lesion Triage Study (ALTS) Group. Interobserver reproducibility of cervical cytologic and histologic interpretations: realistic estimates from the ASCUS-LSIL Triage Study. JAMA 2001;285:1500-5.

10. Ho GY, Bierman R, Beardsley L, et al. Natural history of cervicovaginal papillomavirus infection in young women. $N$ Engl $J$ Med 1998;338:423-8.

11. Sundström K, Lu D, Elfström KM, et al. Follow-up of women with cervical cytological abnormalities showing atypical squamous cells of undetermined significance or low-grade squamous intraepithelial lesion: a nationwide cohort study. Am J Obstet Gynecol 2017;216(1):48.e1-48.e15

12. Pretorius RG, Peterson P, Azizi F, et al. Subsequent risk and presentation of cervical intraepithelial neoplasia $(\mathrm{CIN}) 3$ or cancer after a colposcopic diagnosis of CIN 1 or less. Am J Obstet Gynecol 2006;195:1260-5.

13. Massad LS, Einstein MH, Huh WK, et al. 2012 Updated Consensus Guidelines for the Management of Abnormal Cervical Cancer Screening Tests and Cancer Precursors. Obstetrics \& Gynecology 2013;121:829-46.

14. Bentley J. Executive council of the society of canadian colposcopists; special contributors. Colposcopic management of abnormal cervical cytology and histology. J Obstet Gynaecol Can 2012;34:1188-202.

15. Italian society of colposcopy and cervicovaginal pathology. Guidelines for the management of abnormal pap smear. Società Italiana di Colposcopia e Patologia Cervico Vaginale 2006;1:1-25.

16. Bornstein J, Bentley J, Bösze P, et al. 2011 Colposcopic Terminology of the International Federation for Cervical Pathology and Colposcopy. Obstetrics Gynecol 2012;120:166-72.

17. Katki HA, Gage JC, Schiffman M, et al. Follow-up testing after colposcopy: five-year risk of CIN 2+ after a colposcopic diagnosis of CIN 1 or less. J Low Genit Tract Dis 2013;17(5 Suppl 1):S69-77.

18. Sørbye SW, Arbyn M, Fismen S, et al. HPV E6/E7 mRNA testing is more specific than cytology in post-colposcopy follow-up of women with negative cervical biopsy. PLoS One 2011;6:e26022.

19. Kjær SK, Frederiksen K, Munk C, et al. Long-term absolute risk of cervical intraepithelial neoplasia grade 3 or worse following human papillomavirus infection: role of persistence. J Nat/ Cancer Inst 2010;102:1478-88.

20. Liverani CA, Di Giuseppe J, Clemente N, et al. Length but not transverse diameter of the excision specimen for high-grade cervical intraepithelial neoplasia (CIN 2-3) is a predictor of pregnancy outcome. Eur J Cancer Prev 2016;25:416-22.

21. Sopracordevole F, Clemente N, Delli Carpini G, et al. Trend of decreasing length of cervical cone excision during the last 20 years. Eur Rev Med Pharmacol Sci 2017;21:4747-54.

22. Ciavattini A, Clemente N, Liverani CA, et al. Italian Society of Colposcopy and Cervico-Vaginal Pathology (SICPCV). Cervical Excision Procedure: A Trend of Decreasing Length of Excision Observed in a Multicenter Survey. $J$ Low Genit Tract Dis 2017;21:279-83.

23. Kyrgiou M, Athanasiou A, Paraskevaidi M, et al. Adverse obstetric outcomes after local treatment for cervical preinvasive and early invasive disease according to cone depth: systematic review and meta-analysis. BMJ 2016;354:i3633.

24. Ciavattini A, Clemente N, Delli Carpini G, et al. Loop electrosurgical excision procedure and risk of miscarriage. Fertil Steril 2015;103:1043-8. 